\title{
Electrochemical supercapacitor behaviour of functionalized candle flame carbon soot
}

\author{
C JUSTIN RAJ $^{1}$, BYUNG CHUL KIM${ }^{1}$, BO-BAE CHO ${ }^{1}$, WON-JE CHO ${ }^{1}$, SUNG-JIN KIM ${ }^{2}$, \\ SANG YEUP PARK ${ }^{2}$ and KOOK HYUN YU ${ }^{1, *}$ \\ ${ }^{1}$ Department of Chemistry, Dongguk University-Seoul, Seoul 100 715, Republic of Korea \\ ${ }^{2}$ Department of Ceramic Engineering, Gangneung-Wonju National University, Gangneung 210 702, Republic of Korea
}

MS received 15 March 2015; accepted 17 August 2015

\begin{abstract}
The electrochemical supercapacitor behaviour of bare, washed and nitric acid functionalized candle flame carbon soots were reported. Crystallinity and the morphology of the candle soots were recorded using X-ray diffraction analysis, scanning and transmission electron microscopy, respectively. The nitric acid functionalized candle soot showed an improved Brunauer-Emmett-Teller surface area of 137.93 from $87.495 \mathrm{~m}^{2} \mathrm{~g}^{-1}$ of washed candle soot. The presence of various functional groups in candle soots and the development of oxygen functionalities in the functionalized candle soot were examined through Fourier transform infrared spectroscopy and energy-dispersive X-ray analysis. Raman spectra showed the characteristic peaks corresponding to the D (diamond) and G (graphite) phase of carbon present in the candle soots. The electrochemical characterization was performed by cyclic voltammetry, galvanostatic charge/discharge test and impedance spectroscopy in $1 \mathrm{M} \mathrm{H}_{2} \mathrm{SO}_{4}$ electrolyte. The functionalized candle soot electrode showed an enhanced specific capacitance value of $187 \mathrm{~F} \mathrm{~g}^{-1}$ at $0.15 \mathrm{~A} \mathrm{~g}^{-1}$ discharge current density, which is much higher than that of bare and washed candle soot electrodes.
\end{abstract}

Keywords. Electrode; nanoparticles; carbon soot; functionalized carbon; supercapacitor.

\section{Introduction}

Electrochemical supercapacitor (SC) have attracted considerable attention because of their great potential to meet the demand of both high-energy density and power density in modern energy storage and energy/power back up systems $[1,2]$. Generally, the SCs are classified into two types, namely electrical double layer capacitors (EDLCs), which involve charge separation at electrode-electrolyte interfaces, and pseudocapacitors, which involve faradaic redox reactions that give rise to the pseudocapacitance [2]. Carbon-based materials are most commonly used in SC electrodes fabrication owing to their potential properties including low cost, easy availability, nontoxic nature, environmental friendliness and stability. Various carbon-based nanomaterials such as activated carbon, carbon nanotubes, onion-like carbon, graphene, etc., have been potentially used as a SC electrode materials [3-6]. In carbon-based SC, the surface of carbon electrodes can be modified with few additional pseudocapacitance reactions to enhance the specific capacitance [7,8]. The pseudocapacitance induced by oxygen functionalities in carbon is the most common phenomenon owing to the combination of oxygen with the carbon surface. These oxygen functional groups can be easily introduced on surface of carbon chemically by acid treatment. This kind of treatment is known to generate groups of acidic character, i.e., to impose electron acceptor properties on the carbon surface $[9,10]$. Moreover, the presence

\footnotetext{
*Author for correspondence (yukook@dongguk.edu)
}

of heteroatom on the surface of the carbon nanostructure can determine its surface chemical properties and play a significant role in device applications. The two most important heteroatoms are oxygen and nitrogen, which affect the surface acidic, basic and hydrophilic properties of carbon materials [11-13]. Generally, the oxygen functionalities are introduced to the carbon surface through chemical treatment [14], electrochemical polarization [15], plasma treatment [16] and physical methods [17]. Moreover, the chemical treatment with hot nitric acid is the simplest and reliable method for functionalizing carbon nanomaterials. This nitric acid treatment can significantly enrich the surface functionalities of carbon nanomaterial resulting in enhancement of the surface area and pore volume [7,18].

In general, combustion and thermal decomposition of hydrocarbons have been employed for the synthesis of various types of carbon nanomaterials. The carbon soot is the dominant product of all types of combustion processes and include various novel grades of carbon nanostructures like carbon black [19], fullerenes [20], carbon nanofibres [21], carbon nanotubes [22], etc. The combustion of paraffin wax (candle flame) is also one of the huge sources of carbon soot. This carbon soot consists of about $91.69 \%$ carbon materials with a small amount of hydrogen, nitrogen, oxygen and other insoluble hydrophobic materials. Howard et al [23] reported that the carbon from candle soot was a rich source of carbon nanoparticles such as fullerenes, graphene, nanotubes and nanofibres. Moreover, the carbon nanoparticles in candle soots have excellent optical property like luminescence in the 
visible portion of the spectrum [24-26] and the structural and better quality of graphitization in the candle soot can contribute as a potential material for various modern applications $[27,28]$. In recent times, the candle soot was functionalized with hydroxyl groups to enhance the fluorescent properties of the carbon nanoparticles [29]. The nitric acid functionalized candle soot shows excellent fluorescence property and it was used for bio-imaging application and confirmed that these fluorescent carbon nanoparticles can effectively replaces the toxic cadmium-based quantum dots in bio-imaging [30]. Also, the candle soot surface was modified with $\mathrm{MnO}_{2}$ core shell and used as an electrode material for the fabrication of SC and achieved better electrochemical performance [31]. In this present work, the candle soot was functionalized by nitric acid and the surface state of the candle soot was modified with oxygenated functional groups and used for the fabrication of SC electrode. The electrochemical properties of the electrode was extensively studied using cyclic voltammetry, galvanostatic charge/discharge test and impedance spectroscopy. Furthermore, the structure and morphology of the bare and functionalized candle soots were studied in detail.

\section{Experimental}

\subsection{Materials preparation}

The candle soot was collected by positioning a clean stainless-steel substrate over a paraffin candle flame (yellow region). The as collected candle soot is labelled as BCS (bare candle soot). Then the collected candle soot was washed repeatedly under sonication with acetone, hexane, and deionized water to remove the impurities and unburned hydrocarbons. The washed candle soot (WCS) was dried overnight in hot air oven at $60^{\circ} \mathrm{C}$. For functionalized candle soot (FCS), the washed candle soot was mixed in $1 \mathrm{M}$ nitric acid solution and maintained at $100^{\circ} \mathrm{C}$ for $12 \mathrm{~h}$. Finally, the FCS was removed and washed using deionized water till it reached neutral $\mathrm{pH}$ and dried overnight in a vacuum oven at $60^{\circ} \mathrm{C}$.

\subsection{Electrode fabrication and electrochemical measurements}

The bare (BCS), washed (WCS) and functionalized candle soot (FCS) electrodes were fabricated by mixing the active materials $(70 \mathrm{wt} \%)$, acetylene black $(20 \mathrm{wt} \%)$ and polyvinylidene fluoride (PVDF, $10 \mathrm{wt} \%$ ) in N-methyl-2pyrrolidone (NMP). The obtained paste was coated over a stainless-steel substrate of exposed geometric area $1 \mathrm{~cm}^{2}$ and dried at $100^{\circ} \mathrm{C}$ for $12 \mathrm{~h}$ in a vacuum oven. The mass of the active material present in the electrodes was determined to be $\sim 3 \mathrm{mg}$. Electrochemical studies such as cyclic voltammetry, galvanostatic charge/discharge and impedance measurements were performed at room temperature $\left(\sim 25^{\circ} \mathrm{C}\right)$ using ZIVE-SP2 (Korea) electrochemical workstation in a threeelectrode arrangement, including a working electrode of candle soot, a carbon mesh counter electrode and a $\mathrm{Ag} / \mathrm{AgCl}$ reference electrode in $1 \mathrm{M} \mathrm{H}_{2} \mathrm{SO}_{4}$ aqueous electrolyte. The electrochemical impedance spectra (EIS) of the electrodes were measured in the frequency range of $0.01 \mathrm{~Hz}-100 \mathrm{kHz}$ at an equilibrium open-circuit potential $0 \mathrm{~V}$ with an $\mathrm{AC}$ perturbation of $5 \mathrm{mV}$ in $1 \mathrm{M} \mathrm{H}_{2} \mathrm{SO}_{4}$ electrolyte.

\subsection{Characterization}

The powder X-ray diffraction (XRD) of the candle soots were analysed by XRD, D/Max-2400, Rigaku using a $\mathrm{Cu} \mathrm{K} \alpha$ source operated at $40 \mathrm{kV}$ and $30 \mathrm{~mA}$ in $2 \theta$ range $10-80^{\circ}$. The surface morphology and energy-dispersive X-ray analysis (EDAX) for the samples were carried out by scanning electron microscopy (SEM) (FE-SEM HITACHI model no: S-4700). The transmission electron microscopy (TEM) images were recorded using JEOL (Japan) model JEM2100F. The Fourier transform infrared (FTIR) spectroscopy spectra were recorded with a Perkin Elmer spectrometer by the $\mathrm{KBr}$ pellet technique. The specific surface area was calculated by the Brunauer-Emmett-Teller (BET) technique. The pore size distribution was estimated from the desorption branch of the isotherm by the Barrett-Joyner-Halenda (BJH) method. Raman spectra of samples were measured using a laser Raman microspectrometer using an excitation wavelength of $532 \mathrm{~nm}$.

\section{Results and discussion}

\subsection{Structural and morphological studies}

The XRD spectrum of the candle soots are shown in figure 1 . The samples shown a predominant broad diffraction peak with low intensity, indicating low crystallinity of the sample. The peaks corresponding to the angle $\sim 25^{\circ}$ and $\sim 42^{\circ}$ are assigned to (002) and (100) reflections of crystalline carbon

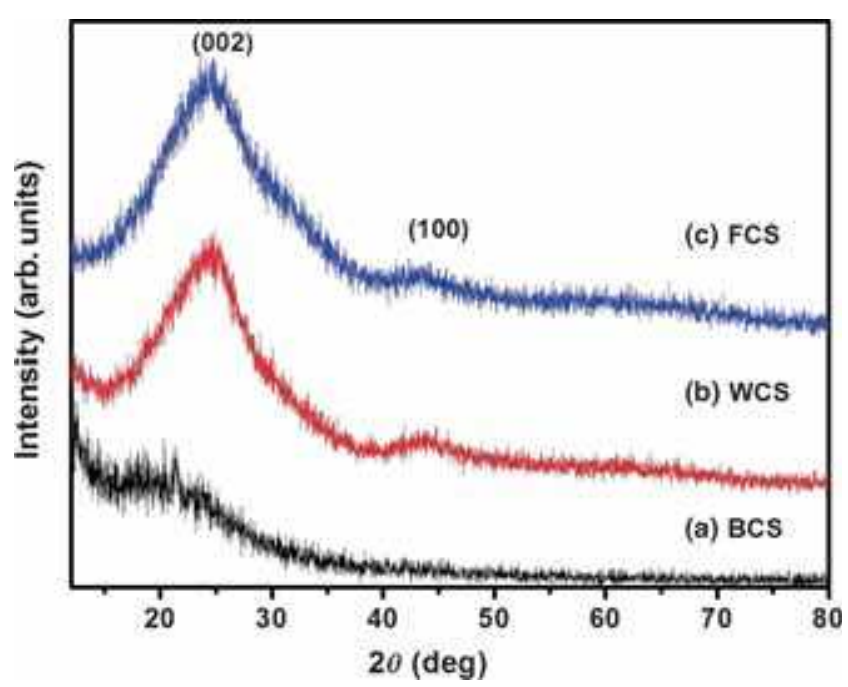

Figure 1. X-ray diffraction pattern of (a) bare, (b) washed and (c) functionalized candle soot. 
nanoparticles (JCPDs \# 41-1487). These suggest that the carbon particles present in the candle soot have some extent of graphitization. But in case of BCS samples shows noisy and low diffraction intensities indicating large amount of amorphous carbon and unburdened wax content present in the sample.

Figure $2 \mathrm{a}$ and $\mathrm{b}$ shows the recorded low- and high-magnification SEM images of as bare candle soot, which clearly displays that the sample is composed of spherical nanoparticles in a form of closely packed aggregates. These aggregates are composed of small nanoparticles ranging from 50 to $100 \mathrm{~nm}$. The SEM images of nitric acid-functionalized electrode are shown in figure $2 \mathrm{c}$ and $\mathrm{d}$. From these images, it is clear that the surface and size of the nanoparticles are significantly altered by the nitric acid treatment. The long duration etching of

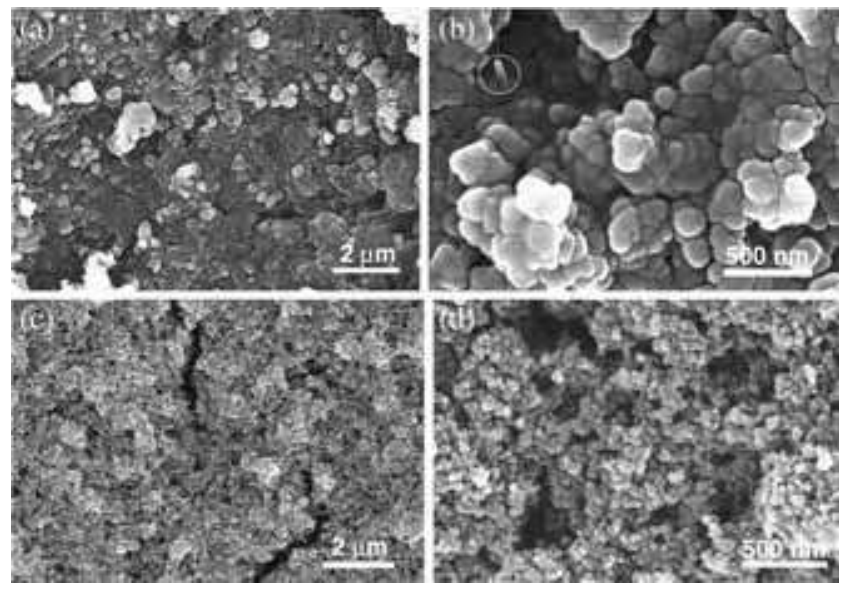

Figure 2. SEM image of (a, b) bare candle soot and (c, d) functionalized candle soot.

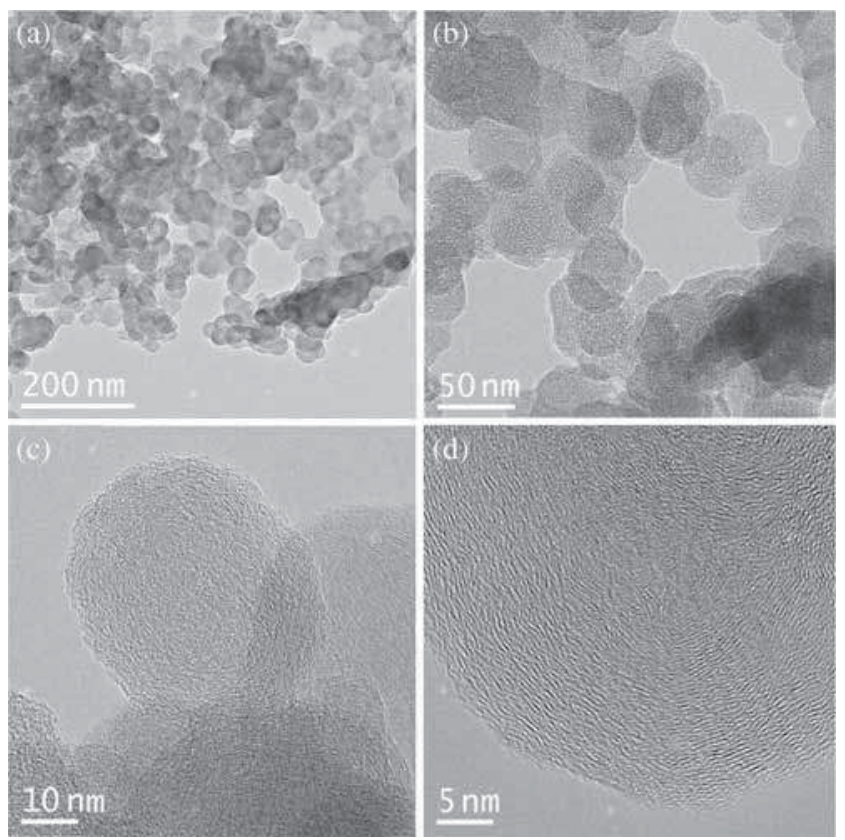

Figure 3. TEM image of (a, b) functionalized candle soot and $(\mathbf{c}, \mathbf{d})$ HRTEM image of functionalized candle soot. nitric acid on the carbon soot leads to reduction of the particle size $(20-50 \mathrm{~nm})$ and improved the porosity of the materials. Furthermore the morphology of the sample was observed clearly using TEM analysis. Figure $3 a$ and $b$ shows the TEM images of FCS sample. From the image it is clearly seen that the sample composed of small chain-linked spherical nanoparticles ranges from $\sim 20$ to $50 \mathrm{~nm}$. The high-resolution TEM images (figure $3 \mathrm{c}$ and $\mathrm{d}$ ) reveal that the candle soot exhibits an onion-like graphitic structure [32].

The specific surface area and pore size distribution of the WCS and FCS were obtained from an analysis of the adsorption/desorption branch of the $\mathrm{N}_{2}$ gas isotherms method. Figure 4 shows that the isotherms are typical for a mesoporous material with a small hysteresis loop at high partial pressures. Also the absorption isotherms shows that the absorption volumes increases when the relative pressure is higher than 0.9 , indicating the presence of mesopores particles with few micropores in both the samples [31]. The BET surface area and corresponding pore volume of the samples were calculated to be $87.495 \mathrm{~m}^{2} \mathrm{~g}^{-1}$ and $0.714 \mathrm{~cm}^{3} \mathrm{~g}^{-1}$ for WCS, and $137.93 \mathrm{~m}^{2} \mathrm{~g}^{-1}$ and $0.627 \mathrm{~m}^{3} \mathrm{~g}^{-1}$ for FCS sample, respectively. As shown by BJH analysis (inset in figure 4), both the samples have a mesopore size distribution of around 32 and $26 \mathrm{~nm}$. From this analysis, it is confirmed that the acid functionalized candle soots effectively improve the surface area due to chemical and morphological changes occurred in the sample [14,18].

Figure 5a shows the FTIR spectrum of BCS, WCS, and FCS samples in the range $400-4000 \mathrm{~cm}^{-1}$. The spectrum of bare carbon soot shows the presence of $\mathrm{CH}_{2}$ asymmetric vibration and $\mathrm{CH}_{3}$ symmetric vibration at 2920 and $2846 \mathrm{~cm}^{-1}$, respectively $[33,34]$ these indicating that the combustion of diolefine in candle soot was partially completed [29]. The sharp absorption doublet of $\mathrm{CO}_{2}$ were observed at 2360 and $2334 \mathrm{~cm}^{-1}$ representing large amount of ash and unburned hydrocarbon present in the candle soot [35]. The spectrum

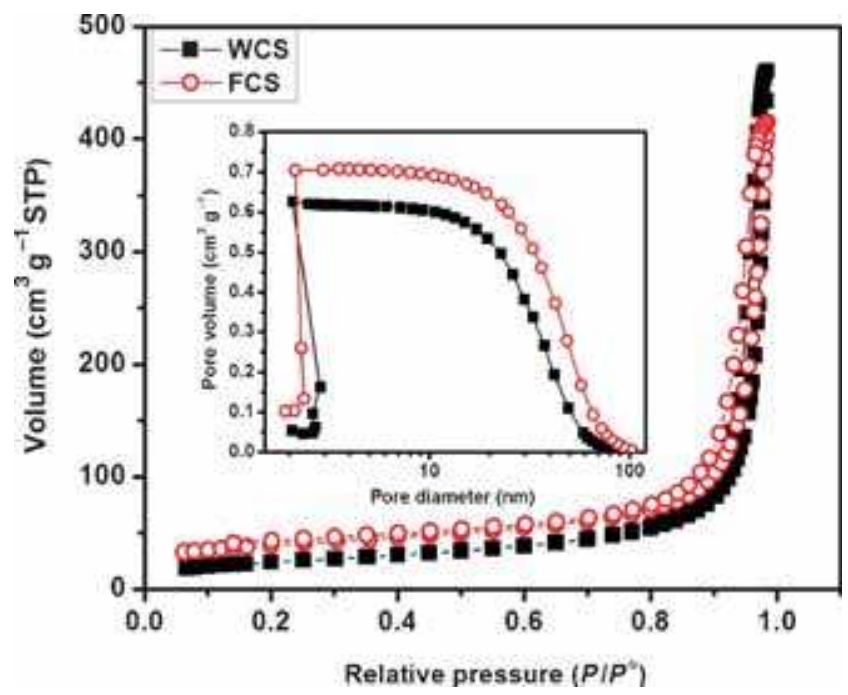

Figure 4. $\mathrm{N}_{2}$ absorption/desorption isotherms and inset pore size distribution of the WCS and FCS samples. 


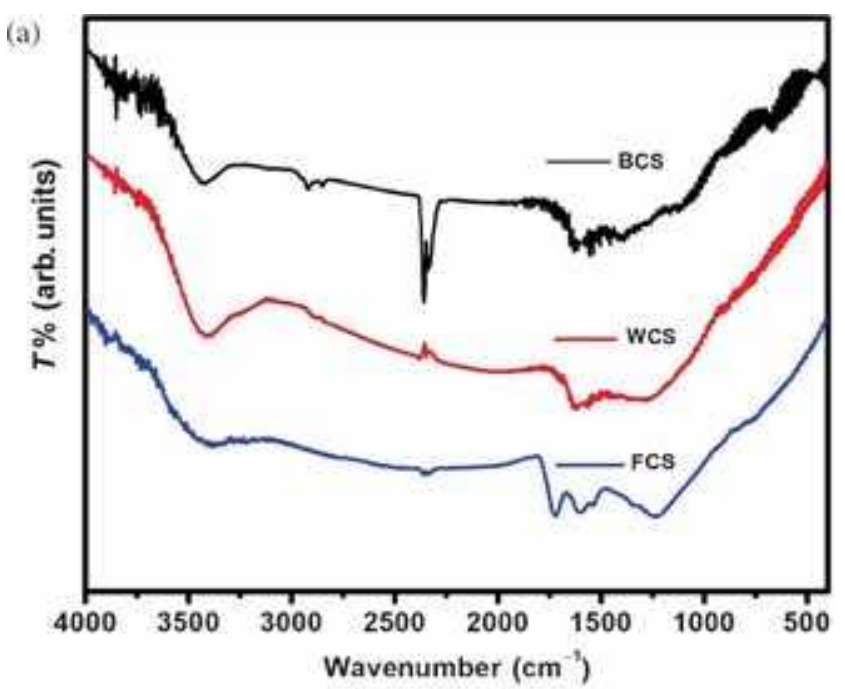

(b)

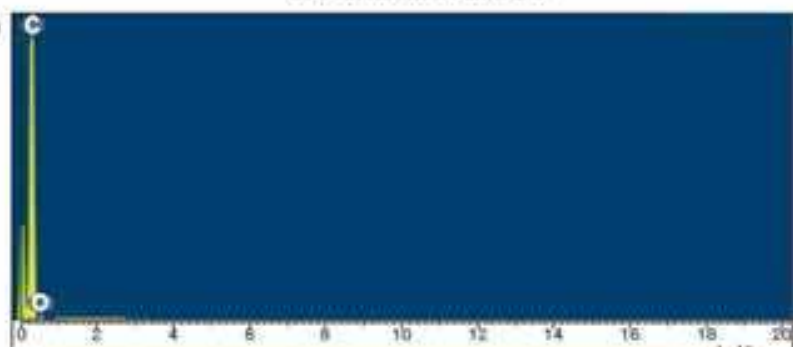

(c)

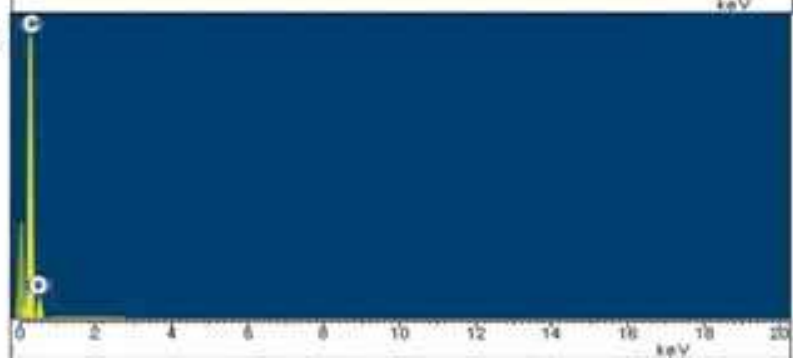

Figure 5. (a) FTIR spectra of bare, washed and functionalized candle soot and EDAX spectra of (b) washed and (c) functionalized candle soot.

of washed samples displays a large decreases in intensity of the above-mentioned peaks representing the improved quality of candle soot. The peak at $3433 \mathrm{~cm}^{-1}$ represents the $\mathrm{OH}$ stretching vibration of hydroxyl group present in the samples. The other low intense absorption peaks at 1615 and $1398 \mathrm{~cm}^{-1}$ similar to the bare candle soot, are due to the presence of aliphatic compounds in candle soot. For functionalized soot, a well-developed band at $1720 \mathrm{~cm}^{-1}$ is attributable to $(\mathrm{C}=\mathrm{O})$ stretching vibration associated with the presence of the carboxylic acid group. A broad band at $1600 \mathrm{~cm}^{-1}$ and a small peak at $1354 \mathrm{~cm}^{-1}$ are due to $(\mathrm{C}=\mathrm{C})$ stretching vibrations. A band at $1250 \mathrm{~cm}^{-1}$ is associated with $(\mathrm{C}-\mathrm{O})$ stretching vibrations [36,37]. These confirm the enhancement of carboxylic functional groups in FCS by nitric acid treatment. The EDAX analysis shows the expected elemental composition, which was carbon and oxygen indicating that the samples free from other impurities (figure $5 \mathrm{~b}$ and c). The WCS sample possess $95.34 \%$ carbon and $4.66 \%$ of oxygen content,

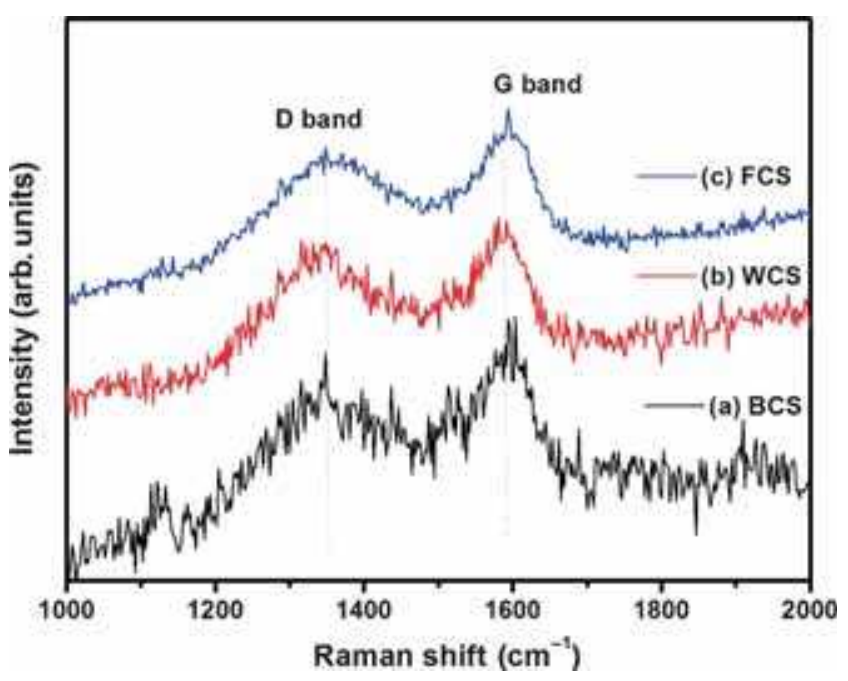

Figure 6. Raman spectra of bare, washed and functionalized candle soot.

in the case of FCS sample consist of $78.37 \%$ of carbon and $21.63 \%$ of oxygen content confirming the enhancement of oxygen functionalities in the nitric acid-treated carbon soot.

Raman spectra of BCS, WCS and FCS samples are shown in figure 6. Two broad peaks are observed at $\sim 1348$ and $\sim 1594 \mathrm{~cm}^{-1}$ corresponding to graphite's D and G bands, respectively. The $\mathrm{G}$ band corresponds to the first-order scattering of the $\mathrm{E}_{2 \mathrm{~g}}$ mode observed for $\mathrm{sp}^{2}$ domains and the $\mathrm{D}$ band is ascribed to $A_{1 g}$ mode which was only observed for disordered graphite [38]. The D band at 1348, 1346 and $1348 \mathrm{~cm}^{-1}$ in BCS, WCS and FCS have been attributed to the presence of amorphous carbon and surface defects. The high intensity the G band at 1595,1593 and $1594 \mathrm{~cm}^{-1}$ of BCS, WCS and FCS are assigned to the graphite phonon mode often associated with graphite, appearing in all the samples suggests that the samples are composed of crystalline graphitic carbon. The relative intensity of these two bands (D and G peaks) which is a measure of disorder in the crystal structure is found to be low $(0.88$; BCS, 0.87; WCS and 0.805 ; FCS) for all the samples $[39,40]$. The little high intensity ratio of BCS shows the high degree of disorder in the carbon sample [41].

\subsection{Cyclic voltammetry study}

Figure $7 \mathrm{a}-\mathrm{c}$ shows the obtained cyclic voltammograms (CVs) of the BCS, WCS, and FCS electrodes, respectively, at various scan rates in $1 \mathrm{M} \mathrm{H}_{2} \mathrm{SO}_{4}$ aqueous electrolyte. At a lower scan rate, CVs of BCS and WCS are close to rectangular shape which resembles the behaviour of EDLC. However, at higher scan rates the CVs deviated from their original rectangular shape to an irregular shape as small amount of oxygenated compounds present on the surface of BCS apparently undergo redox reactions at higher scan rate and result in a pseudocapacitance behaviour with distorted CV curves. In the case of FCS (figure 7c), the curve shows slight tilted rectangular shape indicating that the faradaic process is enhanced in the sample. This pseudocapacitive behaviour can 

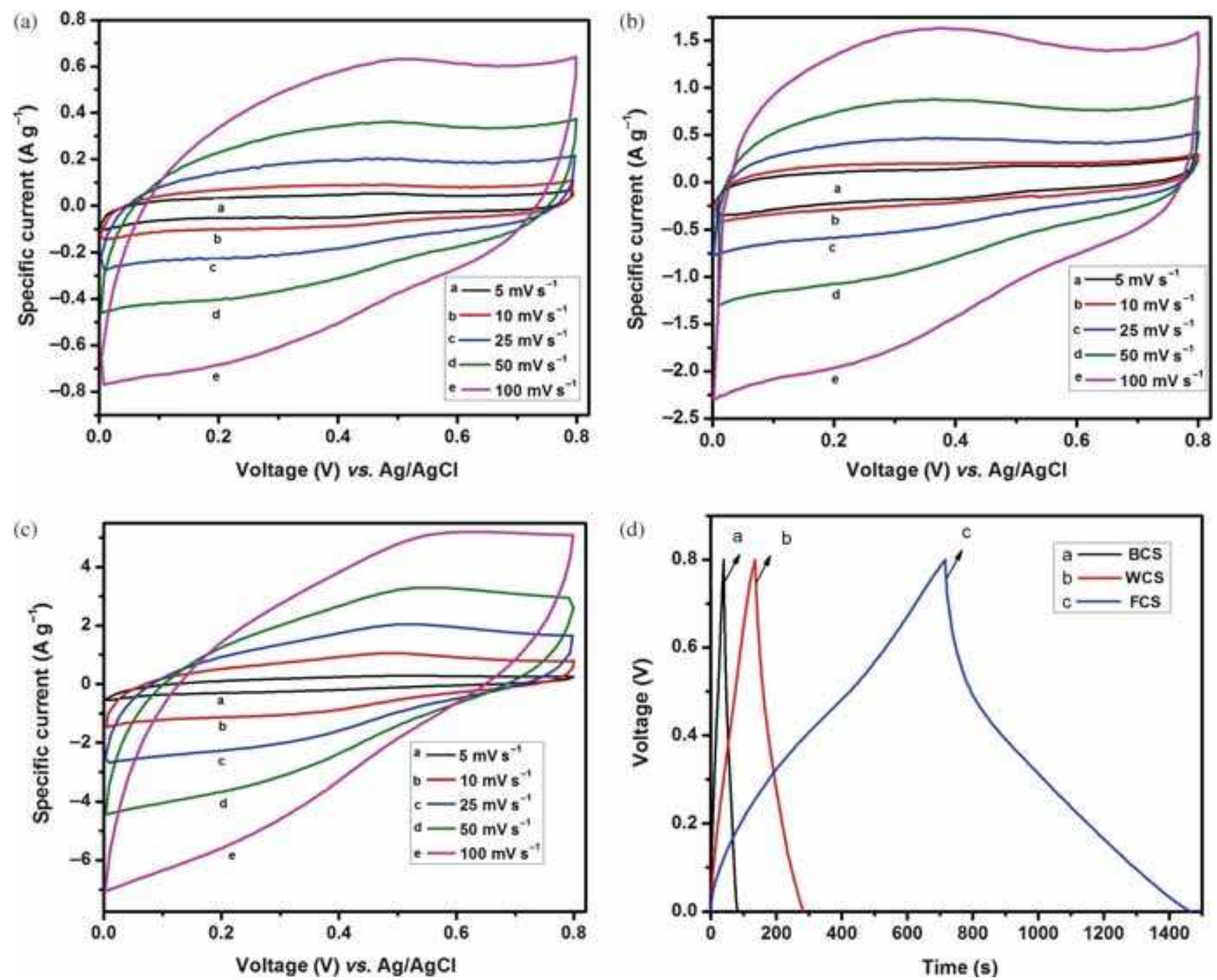

Figure 7. Cyclic voltammograms of electrode (a) BCS, (b) WCS and (c) FCS at various scan rates; and (d) the charge/discharge curves for BCS, WCS and FCS electrodes at a constant current density of $0.15 \mathrm{~A} \mathrm{~g}^{-1}$.

be induced due to excess of oxygenated groups present on the surface of the acid-treated candle soot [42]. From this CVs, the FCS shows a higher background current than that of BCS and WCS electrodes, indicating a higher specific capacitance due to an enhanced faradaic redox reaction.

The specific capacitances $\left(C_{\mathrm{sp}}, \mathrm{F} \mathrm{g}^{-1}\right)$ were calculated from the $\mathrm{CV}$ curves according to

$$
C_{\mathrm{sp}}=\int i \mathrm{~d} V /(2 \times m \times s \times \Delta V),
$$

where $m$ is the mass of active material $(\mathrm{g}), s$ the potential scan rate $\left(\mathrm{mV} \mathrm{s}^{-1}\right), i$ the voltammetric current (A), $\Delta V$ the potential window $(\mathrm{V})$ of $\mathrm{CV}$ curve. The specific capacitance of the FCS was calculated to be $156 \mathrm{~F} \mathrm{~g} \mathrm{~g}^{-1}$ at $5 \mathrm{mV} \mathrm{s}^{-1}$ scan rate, which is much higher than that of WCS $48 \mathrm{~F} \mathrm{~g}^{-1}$ and BCS $16 \mathrm{~F} \mathrm{~g} \mathrm{~g}^{-1}$ at $5 \mathrm{mV} \mathrm{s}^{-1}$. This is mainly attributed to the presence of more oxygenated functional groups in the FCS. These oxygen functionalities result in the occurrence of faradaic peak currents indicating the presence of both
$\mathrm{CO}$ - and $\mathrm{CO}_{2}$-desorbing complexes involved in the electron transfer mechanisms. The redox mechanism for a carbonyl or quinone-type group has been proposed $[42,43]$ as, $>\mathrm{C}_{x} \mathrm{O}$ $+\mathrm{H}^{+}+e^{-} \rightarrow>\mathrm{C}_{x} \mathrm{OH}$, where $>\mathrm{C}_{x} \mathrm{OH}$ represents a phenolor hydroquinone-type complex and $e^{-}$is an electron. This reaction should make partial contribution to the faradaic peak of the FCS electrode. Apart from this, the nitric acid treatment increases the surface area, porosity and reduces the hydrophobic nature of the candle soot which can provide an improvement to the double layer capacitance of the FCS $[44,45]$. The very low specific capacitance value of BCS is mainly due to excess of unburned hydrocarbon and amorphous carbon which reduce the conductivity and increases the hydrophobic property of the sample.

\subsection{Galvanostatic charge/discharge test}

The practical SC characteristics can also be determined through galvanostatic charge/discharge measurements. Figure $7 \mathrm{~d}$ 
shows the typical charge/discharge behaviour of BCS, WCS and FCS electrodes. The electrodes measured at the current density of $0.15 \mathrm{~A} \mathrm{~g}^{-1}$ show nearly triangular form with good reversible charge/discharge cycles. The plot shows that the FCS electrode undertook much longer charge-discharge process than the BCS and WCS. This indicates the larger electrical storage capacity of the FCS electrode than that of other two electrodes. The specific capacitances of the electrodes were determined from the charge/discharge curves by using the following equation:

$$
C_{\mathrm{sp}}=I / m\left(\frac{\mathrm{d} V}{\mathrm{~d} t}\right)
$$

where $I$ is the applied current, $m$ the mass of candle soot in the electrode, and $\mathrm{d} V / \mathrm{d} t$ the slope of the discharge curve. The calculated specific capacitance at the current density of $0.15 \mathrm{~A} \mathrm{~g}^{-1}$ is $187 \mathrm{~F} \mathrm{~g}^{-1}$ for FCS, $51 \mathrm{~F} \mathrm{~g}^{-1}$ for WCS and $16 \mathrm{~F} \mathrm{~g}^{-1}$ for BCS electrodes. These values are nearly consistent with those values obtained from the $\mathrm{CV}$ measurements. Figure 8a presents the variation of specific capacitance with respect to various discharge current densities. It can be seen that the specific capacitance of the electrodes gradually decreases with the increase in discharge current. These decreases in the capacitance can be explained by the reduced diffusion time, since the electrolyte ions cannot be fully accessible to the interior surface of the electrodes for charge storage process at high scan rates [46]. The specific capacitances of FCS were found to be higher than the BCS and WCS electrodes at all discharge current densities. Moreover, at discharge current the plots were found to nearly plateau for both the samples, which reveals the better rate capacity of the candle soot as an electrode material for SC applications.

The specific energy density and the power density of the electrodes were estimated by the respective following equation:

$$
E_{\mathrm{sp}}=1 / 2 \times C_{\mathrm{sp}} V^{2}
$$

and

$$
P=E_{\mathrm{sp}} / \Delta t,
$$

where $V$ is the operating potential, $C_{\mathrm{sp}}$ the specific capacitance of the electrodes and $\Delta t$ the discharge time. The resultant Ragone plots for BCS, WCS, and FCS electrodes are shown in figure $8 \mathrm{~b}$. From the plot, the energy density of FCS increases from 4.82 to $16.63 \mathrm{~W} \mathrm{~h} \mathrm{~kg}^{-1}$ and at the same time the power density decreases from 1591.33 to $80.61 \mathrm{~W} \mathrm{~kg}^{-1}$. The improved energy density of the FCS electrode can be attributed to the pseudocapacitance induced by the oxygenated functional groups. The decrement in power density of the FCS electrode is because of the increased internal resistance of the electrode which in turn is caused by the enhanced oxygenated functional groups in the electrode. The cycling life and the deliverable energy efficiency of the BCS, WCS and FCS electrodes were estimated by performing continuous charge/discharge cycles (1000 cycles) at a constant
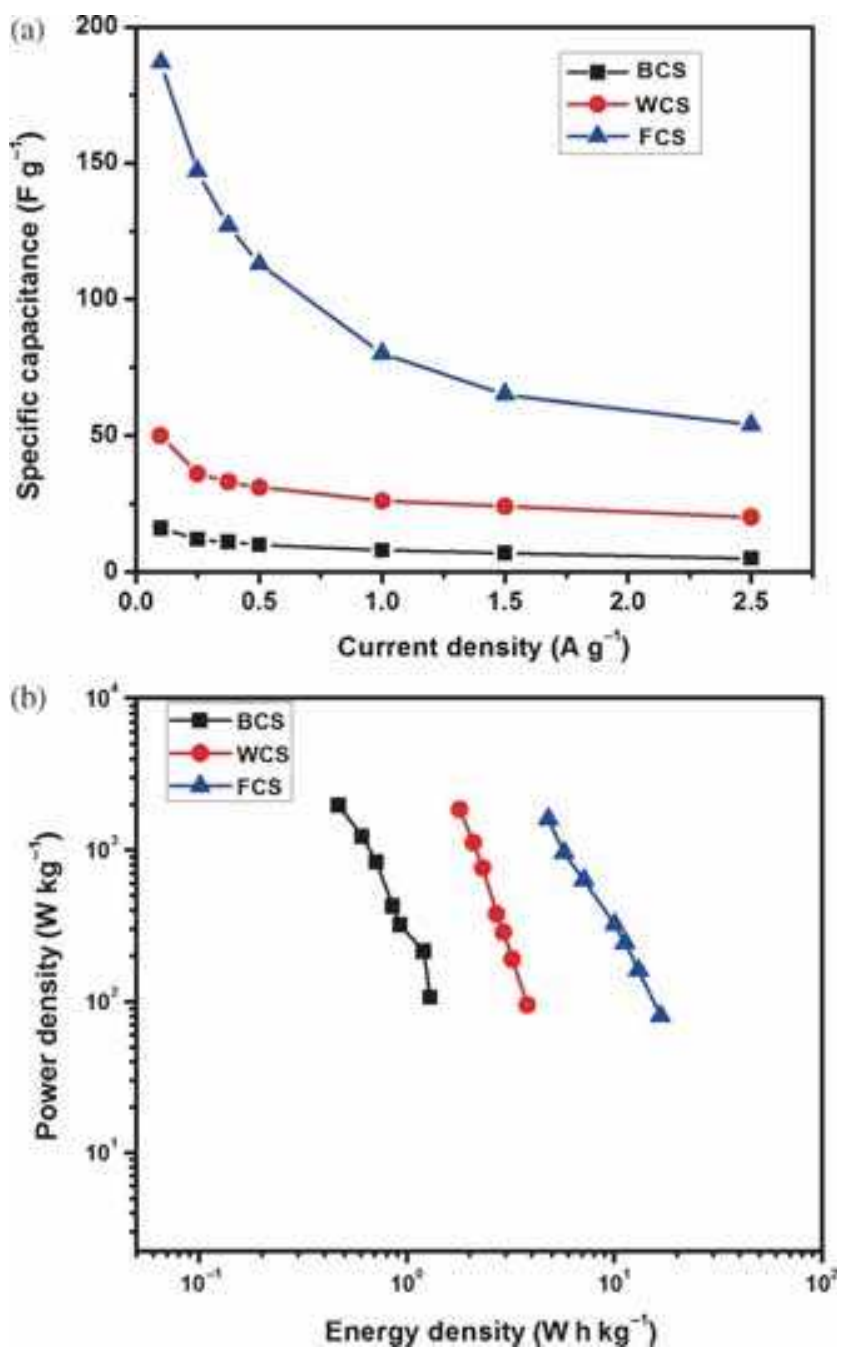

Figure 8. (a) Variation of specific capacitance with scan rate for BCS, WCS and FCS electrodes and (b) Ragone plots of BCS, WCS and FCS electrodes.

discharge current density of $1 \mathrm{~A} \mathrm{~g}^{-1}$ and calculated using the equation

$$
\eta=t_{\mathrm{d}} / t_{\mathrm{c}} \times 100 \%
$$

where $t_{\mathrm{d}}$ and $t_{\mathrm{c}}$ are the discharge and charge times, respectively. The calculated values of $\eta$ after 1000th cycle for BCS, WCS and FCS electrodes are 86.18, 99.2 and 98.32\%. The loss in capacitance of FCS electrode after 1000th cycle is nearly $1.68 \%$, which confirms the better stability of the electrode in the electrolyte with a good deliverable energy efficiency.

\subsection{Impedance spectroscopy}

Electrochemical impedance spectroscopy (EIS) analysis was performed to study the resistive and capacitive behaviour of the electrodes. The obtained Nyquist plot for the electrodes 
in the frequency range between $0.01 \mathrm{~Hz}$ and $100 \mathrm{kHz}$ is shown in figure 9. Nyquist plots are composed of a spike in the low-frequency region and an incomplete semicircle in the high-frequency region. The slope of the low-frequency spike is close to $90^{\circ}$ along the imaginary impedance axis, indicating a pronounced capacitive behaviour while the highfrequency semicircle represents the high interfacial charge transfer resistance due to active materials conductivity and surface states [47-49]. The impedance spectra were analysed by Zview software on the basis of the electrical equivalent circuit, as shown in inset of figure 9 . The bulk solution resistance is represented as $R_{\mathrm{S}}$, a constant phase element $\mathrm{CPE}_{1}$ representing double layer capacitance was connected parallel with the charge transfer resistance $R_{\mathrm{ct}}$ is modelling with the high-frequency semicircles, and a constant phase element $\left(\mathrm{CPE}_{2}\right)$ is connected in series with the resistance $R_{\mathrm{ct}}$ which represents the pseudocapacitance associated with the electrode. The overall resistance of the electrodes is the combination of $R_{\mathrm{s}}$ with $R_{\mathrm{ct}}$ values and this result was found to be much higher for the BCS electrode $(41.63 \Omega)$ compared with WCS $(2.34 \Omega)$. This reveals that the large particles of carbon aggregates and the hydrophobic nature of bare candle soot can restrict the easy ion migration into the pores of electrode exhibiting a high internal resistance in the electrode $[50,51]$. The overall resistance of FCS $(8.52 \Omega)$ is found to be higher than WCS, which may be due to the existence of large oxygenated groups present in the sample as well as the inert nature of the carbon surface. From the fitted data the pseudocapacitance associated with the three electrodes are 0.008 , 0.0022 and $0.044 \mathrm{~F}$ for BCS, WCS and FCS, respectively. This observation confirms that the high value of specific capacitance for FCS originated from the additional faradaic reaction of the oxygenated functional group present in the sample.

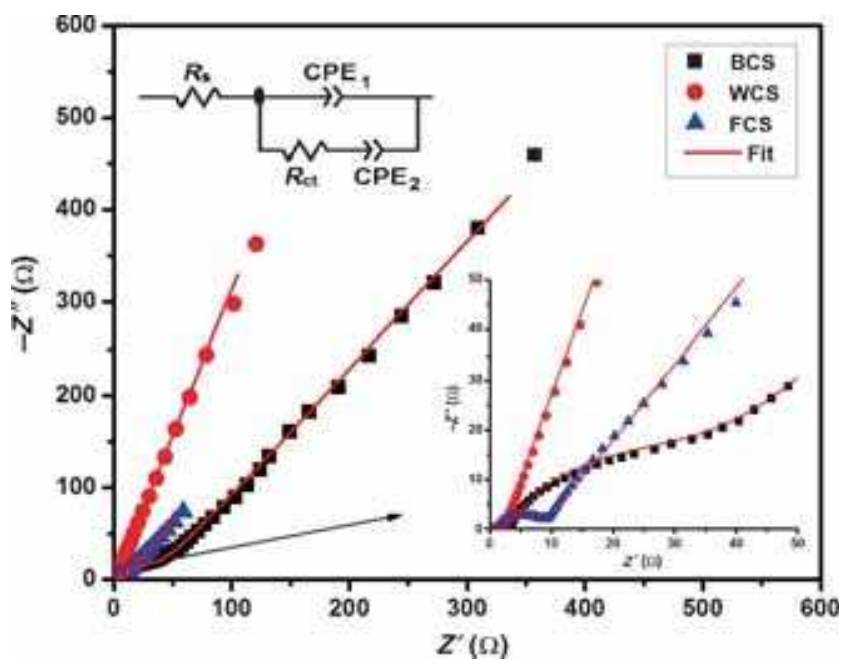

Figure 9. Nyquist plots for the BCS, WCS and FCS electrodes, inset shows the equivalent circuit of fit and the magnified portion of high-frequency region of Nyquist plots.

\section{Conclusion}

SC electrodes were fabricated by employing bare, washed, and nitric acid functionalized candle flame soot. The fabricated candle soot electrodes show the existence of crystalline carbon nanoparticles with an excellent surface morphology. The functionalized carbon soot showed an improved BET surface area of 137.93 from $87.495 \mathrm{~m}^{2} \mathrm{~g}^{-1}$ than that of washed candle soot. The presence of enhanced oxygen functional groups in the functionalized candle soot was confirmed through FTIR spectroscopy. The electrochemical analysis of the functionalized candle soot showed a specific capacitance of $187 \mathrm{~F} \mathrm{~g}^{-1}$ at $0.15 \mathrm{~A} \mathrm{~g}^{-1}$ current density, high specific energy density $16.63 \mathrm{~W} \mathrm{~h} \mathrm{~kg}^{-1}$, and a low internal resistance applicable for SC electrodes. The improved surface area and the large pseudocapacitance induced by oxygen functional groups were responsible for the enhancement in overall electrochemical properties of the functionalized candle soot electrode.

\section{Acknowledgements}

We would like to acknowledge authorities of Dongguk University-Seoul for their moral and financial support. This research was supported by National Research Foundation of South Korea (NRF) (no. 2014030484).

\section{References}

[1] Nishino A 1996 J. Power Sources 60137

[2] Burke A 2000 J. Power Sources 9137

[3] Zhao Q, Wang X, Liu J, Wang H, Zhang Y, Gao J, Liu J and Lu Q 2015 J. Electrochem. Soc. 162 A845

[4] Jiang H, Lee P S and Li C 2013 Energy Environ. Sci. 641

[5] Wang G, Wang L and Zhang J 2012 Chem. Soc. Rev. 41797

[6] Wang Q, Cao Q, Wang X, Jing B, Kuang H and Zhou L 2013 J. Solid State Electrochem. 172731

[7] Frackowiak E and Beguin F 2001 Carbon 39937

[8] Qu D 2002 J. Power Sources 109403

[9] Biniak S, Swiaztkowski A and Pakula M 2001 Radovic L R (ed.) Chemistry and physics of carbon (New York: Marcel Dekker)

[10] Laszlo K, Tombacz E and Josepovits K 2001 Carbon 391217

[11] Kim B K, Ryu S K, Kim B J and Park S J 2006 J. Colloid Interface Sci. 302695

[12] Oda H, Yamashita A, Minoura S, Okamoto M and Morimoto T 2006 J. Power Sources 1581510

[13] El-Sayed Y and Bandosz T J 2005 Langmuir 211282

[14] Du J X, Mishra D and Ting J M 2013 Appl. Surf. Sci. 285483

[15] Momma T, Liu X, Osaka T, Ushio Y and Sawada Y 1996 J. Power Sources 60249

[16] Ishikawa M, Sakamoto A, Morita M, Matsuda Y and Ishida K 1996 J. Power Sources 60233

[17] Koresh J and Soffer A 1977 J. Electrochem. Soc. 1241379

[18] Lakshminarayanan P V, Toghiani H and Pittman C U 2004 Carbon 422433 
[19] Surovikin V F, Rogov A V and Vershinin L V 1975 Combust. Explos. Shock Waves 11202

[20] Howard J B, Lafleur A L, Makarovsky Y, Mitra S, Pope C J and Yadav T K 1992 Carbon 301183

[21] Li F, Zou X P, Cheng J, Zhang H, Ren P, Wang M and Zhu G 2008 J. Cent. South Univ. Technol. 1515

[22] Pereira J S F, Antes F G, Diehl L O, Knorr C L, Mortari S R, Dressler V L and Flores E M M 2010 J. Anal. At. Spectrom. 251268

[23] Howard J B, MAcKinnon J T, Makarovsky Y, Lafleur A L and Johnson M E 1991 Nature 352139

[24] Liu H, Ye T and Mao C 2007 Angew Chem. Int. Ed. 46 6473

[25] Kumar P and Bohidar H B 2012 J. Nanopart. Res. 14 948

[26] Bottini M and Mustelin T 2007 Nat. Nanotechnol. 2599

[27] Deng X, Mammen L, Butt H J and Vollmer D 2012 Science 33567

[28] Su Z, Zhou W and Zhang Y 2011 Chem. Commun. 474700

[29] Khanam A, Tripathi S K, Roy D and Nasim M 2013 Colloids Surf. B 10263

[30] Ray S C, Saha A, Jana N R and Sarkar R 2009 J. Phys. Chem. $C 11318546$

[31] Zhang B, Wang D, Yu B, Zhou F and Liu W 2014 RSC Adv. 4 2586

[32] Su Z, Zhou W and Zhang Y 2011 Chem. Commun. 474700

[33] Lin T, Chen J, Bi H, Wan D, Huang F, Xie X and Jiang M 2013 J. Mater. Chem. A 1500

[34] Mohan A N and Manoj B 2012 Int. J. Electrochem. Sci. 7 9537

[35] Yamada H and Person W B 1964 J. Chem. Phys. 412478
[36] Kirchner U, Scheer V and Vogt R 2000 J. Phys. Chem. A 104 8908

[37] Dubey P, Muthukumar D, Dash S, Mukopadhyay R and Sarkar S 2005 Pramana J. Phys. 65681

[38] Ferrari A C and Robertson J 2000 Phys. Rev. B 6114095

[39] Muller M, Maultzsch J, Wunderlich D, Hirsch A and Thomsen C 2007 Phys. Status Solidi 2444056

[40] Shooto N D and Dikio E D 2012 Int. J. Electrochem. Sci. 7 4335

[41] Roh J S 2008 Carbon Lett. 9127

[42] Nian Y R and Teng H 2002 J. Electrochem. Soc. 149 A1008

[43] Conway B E 1999 Electrochemical supercapacitors (New York: Kluwer Academic/Plenum Publishers) p 259

[44] Shan J, Liu X, Cui H F, Zhang W D, Sheu F S and Lim T M 2005 Electrochem. Commun. 7249

[45] Xiao X, Li T, Peng Z, Jin H, Zhong Q, Hu Q, Yao B, Luo Q, Zhang C, Gong L, Chen J, Gogotsi Y and Zhou J 2014 Nano Energy 61

[46] Wang J G, Yang Y, Huang Z H and Kang F 2013 Carbon 61 190

[47] Huang Y, Candelaria S L, Li Y, Li Z, Tian J, Zhang L and Cao G 2014 J. Power Sources 25290

[48] Sugimoto W, Iwata H, Yokoshima K, Murakami Y and Takasu Y 2005 J. Phys. Chem. B $\mathbf{1 0 9} 7330$

[49] Xiong W, Liu M X, Gan L H, Lv Y K, Li Y, Yang L, Xu Z J, Hao Z X, Liu H L and Chen L W 2011 J. Power Sources 196 10461

[50] Chen W C, Wen T C and Teng H 2003 Electrochim. Acta 48 641

[51] Lei C, Markoulidis F, Ashitaka Z and Lekakou C 2013 Electrochim. Acta 92183 